\title{
The magnetically-active, low-mass, triple system WDS 19312+3607 (Research Note)
}

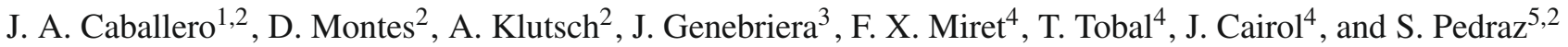 \\ 1 Centro de Astrobiología (CSIC-INTA), Carretera de Ajalvir km 4, 28850 Torrejón de Ardoz, Madrid, Spain \\ e-mail: caballero@astrax.fis.ucm.es \\ 2 Departamento de Astrofísica y Ciencias de la Atmósfera, Facultad de Física, Universidad Complutense de Madrid, 28040 Madrid, \\ Spain \\ 3 Observatorio de Tacande, La Palma, Spain \\ 4 Observatori Astronòmic del Garraf, Barcelona, Spain \\ 5 Centro Astronómico Hispano Alemán de Calar Alto (CSIC-MPG), c/ Jesús Durbán Remón 2-2, 04004 Almería, Spain
}

Received 12 March 2010 / Accepted 7 June 2010

\section{ABSTRACT}

\begin{abstract}
Aims. We investigated in detail the system WDS $19312+3607$, whose primary is an active M4.5Ve star previously inferred to be young $(\tau \sim 300-500 \mathrm{Ma})$ based on its high X-ray luminosity.

Methods. We collected intermediate- and low-resolution optical spectra taken with $2 \mathrm{~m}$-class telescopes, photometric data from the $B$ to $8 \mu \mathrm{m}$ bands, and data for eleven astrometric epochs with a time baseline of over 56 years for the two components in the system, G $125-15$ and G $125-14$

Results. We derived the M4.5V spectral types of both stars, confirmed their common proper motion, estimated their heliocentric distance and projected physical separation, determined their Galactocentric space velocities, and deduced a most-probable age of older than $600 \mathrm{Ma}$. We discovered that the primary, G 125-15, is an inflated, double-lined, spectroscopic binary with a short period of photometric variability of $P \sim 1.6 \mathrm{~d}$, which we associated with orbital synchronisation. The observed X-ray and $\mathrm{H} \alpha$ emissions, photometric variability, and abnormal radius and effective temperature of G 125-15 AB are indicative of strong magnetic activity, possibly because of the rapid rotation. In addition, the estimated projected physical separation between G $125-15$ AB and G $125-14$ of about $1200 \mathrm{AU}$ ensures that WDS $19312+3607$ is one of the widest systems with intermediate M-type primaries.

Conclusions. $\mathrm{G} 125-15 \mathrm{AB}$ is a nearby $(d \approx 26 \mathrm{pc})$, bright $(J \approx 9.6 \mathrm{mag})$, active spectroscopic binary with a single proper-motion companion of the same spectral type at a wide separation. They are thus ideal targets for specific follow-ups to investigate wide and close multiplicity or stellar expansion and surface cooling because of the lower convective efficiency.
\end{abstract}

Key words. stars: activity - binaries: visual - binaries: spectroscopic - stars: individual: G 125-14 - stars: individual: G 125-15 stars: low mass

\section{Introduction}

The binary WDS 19312+3607 (Washington Double Star identifier: GIC 158) is formed by the two nearby high proper-motion stars G 125-15 and G 125-14 (Giclas et al. 1971; Worley \& Douglass 1997; Caballero et al. 2010). The primary, G 125-15, is an active M4.5Ve star with near-solar metal abundance (Reid et al. 2004). The secondary, G 125-14, is about 1 mag fainter in the visible and has never been investigated spectroscopically.

Interestingly, the system WDS 19312+3607 was hypothesised to be a few hundred million years old. Fuhrmeister \& Schmitt (2003) associated a ROSAT soft X-ray source with G 125-15. Daemgen et al. (2007) and Allen \& Reid (2008) inferred from its location in a $\log \left(F_{\mathrm{X}} / F_{J}\right)$ versus $V-J$ diagram that $\mathrm{G} \mathrm{125-15}$ has X-ray activity levels that exceed those of Pleiades stars of a similar spectral type and conservatively assumed an age of 300-500 Ma, although the M dwarfs in their sample may be younger. Youth, closeness, and late spectral type are optimal properties when searching for faint companions to stars, ensuring that G 125-15 became the target of adaptive optics and IRAC/Spitzer searches by Daemgen et al. (2007) and Allen \& Reid (2008), respectively. They provided restrictive upper limits to the magnitudes and masses of hypothetical brown dwarf and planetary companions at close separations (up to a few arcseconds). The secondary star, G 125-14, fell out of the field of view of Altair+NIRI/Gemini North in Daemgen et al. (2007), but is among the brightest sources in the IRAC/Spitzer images in Allen \& Reid (2008). Both groups unintentionally overlooked the existence of the stellar companion. They did not take into account the photometric variability of the primary either, which might be related to activity (and in turn to youth). During the Hungarian Automated Telescope Network (HATnet) variability survey in a field chosen to overlap with the Kepler mission, Hartman et al. (2004) found G 125-15 to be a periodic variable with a pulsating variable-like light curve. They measured a period $P_{\text {phot }}=1.6267 \mathrm{~d}$ and an amplitude $\Delta I=0.097 \mathrm{mag}$. The secondary star, G 125-14, was not analysed.

From the approximate angular separation of $47 \mathrm{arcsec}$ between G 125-15 and G 125-14 and preliminary estimates of the heliocentric distance to the primary based on spectroscopic parallax ( $d \sim 15$ pc - Reid et al. 2004; Allen \& Reid 2008), we derived a rough projected physical separation $s \sim 700 \mathrm{AU}$. This wide separation and the late spectral type of the primary would make the system one of the widest low-mass binaries in the field (Caballero 2007, 2009; Artigau et al. 2007; Radigan et al. 2009). If the age estimation by Daemgen et al. (2007) and Allen \& Reid (2008) were correct, the WDS $19312+3607$ system would be the first young wide low-mass binary in the solar neighbourhood. 
Table 1. Multi-epoch astrometric measurements of WDS 19312+3607.

\begin{tabular}{lccl}
\hline \hline Epoch & $\begin{array}{c}\rho \\
{[\operatorname{arcsec}]}\end{array}$ & $\begin{array}{c}\theta \\
{[\mathrm{deg}]}\end{array}$ & Source $^{a}$ \\
\hline 1952 Jul. 17 & $45.5 \pm 0.4$ & 348 & POSS-I Red \\
1988 Jun. 13 & $46.2 \pm 0.4$ & 347 & POSS-II Blue \\
1992 Aug. 31 & $46.0 \pm 0.4$ & 348 & POSS-II Red \\
1993 Jun. 13 & $45.7 \pm 0.4$ & 347 & POSS-II Infrared \\
1994 Aug. 20 & $45.82 \pm 0.10$ & 347.3 & SDSS DR7 \\
1998 Jun. 01 & $45.80 \pm 0.12$ & 347.4 & 2MASS \\
2001 Aug. 26 & $45.90 \pm 0.06$ & 347.5 & CMC14 \\
2004 Oct. 30 & $45.81 \pm 0.10$ & 347.4 & IRAC \\
2008 Mar. 07 & $45.82 \pm 0.15$ & 347.3 & Tacande \\
2008 May 05 & $45.70 \pm 0.15$ & 347.4 & CAFOS \\
2008 Oct. 23 & $45.80 \pm 0.15$ & 347.3 & Tacande \\
\hline
\end{tabular}

Notes. ${ }^{(a)}$ POSS: SuperCOSMOS (Hambly et al. 2001) digitisations of the First (1948-1958) and Second (1985-2000) Palomar Observatory Sky Survey; 2MASS: Two-Micron All-Sky Survey (Skrutskie et al. 2006); SDSS DR7: Sloan Digital Sky Survey (Abazajian et al. 2009); CMC14: Carlsberg Meridian Catalog 14 (Muiños 2006); IRAC: Spitzer Heritage Archive, program name/identification INR/3286; CAFOS: Calar Alto Faint Object Spectrograph with the Site\#1d_15 detector at the $2.2 \mathrm{~m}$ Calar Alto Teleskop in Almería, Spain; Tacande: dual CCD camera SBIG ST-8XME at the $0.4 \mathrm{~m}$ Telescopio del Observatorio de Tacande in La Palma, Spain.

Thus, we aimed to characterise this system in detail with new observations and data compilation from the literature.

\section{Observations and analysis}

We first used 11 astrometric epochs to measure the mean angular separation, position angle, and common proper motion of G 125-15 and G 125-14, as listed in Table 1. We collected coordinates tabulated by the SDSS DR7, 2MASS, and CMC14 catalogues, and carried out standard astrometric analyses on public images (POSS, IRAC) and optical images obatined by us with CAFOS/2.2 $\mathrm{m} \mathrm{Calar} \mathrm{Alto}^{1}$ and $0.4 \mathrm{~m} \mathrm{Tacande}^{2}$. All the measurements are consistent within $1 \sigma$ with a mean angular separation $\bar{\rho}=45.83 \pm 0.17 \mathrm{arcsec}$ and a position angle $\bar{\theta}=347.5 \pm 0.4 \mathrm{deg}$. For comparison, during the 56.271 years of our time baseline, the two stars travelled together by about 10.3 arcsec. Using the methodology presented in Caballero (2010), we determined the proper motion of the primary at $\left(\mu_{\alpha} \cos \delta, \mu_{\delta}\right)=(-116.3 \pm$ $2.0,-100.6 \pm 1.2)$ mas $\mathrm{a}^{-1}$, which supersedes previous determinations with larger uncertainties (Luyten 1979; Salim \& Gould 2003; Hanson et al. 2004; Lépine \& Shara 2005; Ivanov 2008).

We next compiled BVRI, ugriz, JHK $K_{\mathrm{s}}$, and [3.6] [4.5][5.8][8.0] photometric data of $G$ 125-15 and G 125-14, which are listed in Table 2 with their associated uncertainties. CAFOS images in the BVRI bands were calibrated using stars in common with a number of overlapping optical catalogues (Høg et al. 2000; Weis 1996; Hartman et al. 2004). We retrieved ugriz and $J H K_{\mathrm{s}}$ magnitudes and coordinates from the SDSS and 2MASS catalogues, respectively (the SDSS $i z$ magnitudes of G 125-15 were affected by saturation). The magnitudes of G 125-15 in the four IRAC/Spitzer channels were taken from Allen \& Reid (2008), while those of G 125-14 were measured by us on the public IRAC post-calibrated images.

\footnotetext{
1 http://wwW. caha.es/alises/cafos/cafos.html

2 http://www . astropalma.com/astropalma_eng.htm
}

Table 2. Basic data of G 125-15 AB and G 125-14.

\begin{tabular}{lcc}
\hline \hline & $\mathrm{G} 125-15 \mathrm{AB}$ & $\mathrm{G} 125-14$ \\
\hline$\alpha^{\text {J2000 }}$ & 193112.57 & 193111.75 \\
$\delta^{\text {J2000 }}$ & +360730.1 & +360814.8 \\
$u[\mathrm{mag}]$ & $17.094 \pm 0.009$ & $18.598 \pm 0.020$ \\
$B$ [mag] & $15.61 \pm 0.05$ & $16.58 \pm 0.06$ \\
$g$ [mag] & $15.075 \pm 0.003$ & $16.072 \pm 0.003$ \\
$V[\mathrm{mag}]$ & $14.12 \pm 0.09$ & $15.16 \pm 0.10$ \\
$r[\mathrm{mag}]$ & $14.148 \pm 0.010$ & $14.690 \pm 0.003$ \\
$R[\mathrm{mag}]$ & $12.60 \pm 0.06$ & $13.71 \pm 0.06$ \\
$i[\mathrm{mag}]$ & $\ldots$ & $13.852 \pm 0.010$ \\
$I[\mathrm{mag}]$ & $11.02 \pm 0.05$ & $12.30 \pm 0.05$ \\
$z[\mathrm{mag}]$ & $\ldots$ & $13.327 \pm 0.003$ \\
$J[\mathrm{mag}]$ & $9.609 \pm 0.022$ & $10.924 \pm 0.022$ \\
$H[\mathrm{mag}]$ & $9.061 \pm 0.020$ & $10.408 \pm 0.020$ \\
$K_{\mathrm{s}}[\mathrm{mag}]$ & $8.839 \pm 0.019$ & $10.137 \pm 0.019$ \\
{$[3.6][\mathrm{mag}]$} & $8.9 \pm 0.2$ & $10.0 \pm 0.2$ \\
{$[4.5][\mathrm{mag}]$} & $8.59 \pm 0.01$ & $9.90 \pm 0.05$ \\
{$[5.8][\mathrm{mag}]$} & $8.45 \pm 0.01$ & $9.79 \pm 0.05$ \\
{$[8.0][\mathrm{mag}]$} & $8.39 \pm 0.01$ & $9.71 \pm 0.05$ \\
$\mathrm{Sp} . \mathrm{type}$ & $\mathrm{M} 4.5 \pm 0.5 \mathrm{Ve}$ & $\mathrm{M} 4.5 \pm 0.5 \mathrm{~V}$ \\
$V_{r}\left[\mathrm{~km} \mathrm{~s}{ }^{-1}\right]$ & $-23 \pm 5^{a}$ & $-26 \pm 2$ \\
$\mathrm{p} E W(\mathrm{H} \alpha)[\AA]$ & $-5.8 \pm 0.7$ & $<+0.13$ \\
$\mathrm{p} E W(\mathrm{Li} \mathrm{I})[\AA]$ & $<+0.13$ & $<+0.13$ \\
$M_{J}[\mathrm{mag}]$ & $7.5_{-0.8}^{+0.7}$ & $8.8_{-0.8}^{+0.7}$ \\
$\mathcal{M}\left[M_{\odot}\right]$ & $0.18 \pm 0.06 / 0.18 \pm 0.06$ & $0.18 \pm 0.05$ \\
\hline & &
\end{tabular}

Notes. ${ }^{(a)}$ We tabulate the mean radial velocity of G $125-15 \mathrm{AB}$. The individual values were $-43 \pm 5$ and $-3 \pm 5 \mathrm{~km} \mathrm{~s}^{-1}$ for the components $\mathrm{A}$ and $\mathrm{B}$, respectively.

On 2008 May 05, we used CAFOS with the grism Blue-400 to acquire low-resolution optical spectra $(R \sim 200$ at $\mathrm{H} \alpha$ $\lambda 6562.8 \AA$ ) of both G $125-15$ and G 125-14. The two stars were imaged simultaneously in the long slit (i.e., we did not observe in parallactic angle). We also observed the late-type dwarf FL Vir AB (M5.5Ve; Joy 1947) and the spectrophotometric standard star HZ 44. We carried out the bias correction, flat-fielding, spectra extraction, wavelength calibration, and instrumental response correction following standard procedures within the astronomical data reduction package $\mathrm{RED}_{\mathrm{m}}^{\mathrm{uc}} \mathrm{E}$ (Cardiel 1999) ${ }^{3}$. Our useful wavelength coverage was from $4000 \AA$ to $10000 \AA$. The final CAFOS spectra of G 125-15, G 125-14, and FL Vir AB are shown in Fig. 1. From our data and classification based on pseudo-continuum indices (e.g., Martín et al. 1999), we agreed with the spectral type determination of the primary of M4.5Ve by Reid et al. (2004). The spectral types of G 125-15 and G 125-14 are identical within an uncertainty of 0.5 dex (Table 2).

Two new spectra were taken on 2009 Sep. 09 with the Intermediate Dispersion Spectrograph (IDS) ${ }^{4}$ at the $2.5 \mathrm{~m}$ Isaac Newton Telescope (INT) on the Observatorio del Roque de los Muchachos, La Palma, Spain. In this case, we used the H1800V grating and the 0.95 arcsec slit, which provided a spectral resolution power of $R \sim 9200$, and observed in parallactic angle. With the same configuration, we also obtained spectra of the comparison stars GJ 687 (M3.5V) and GJ 1227 (M4.5V) and a number of radial-velocity standards. The reduction and analysis of the

\footnotetext{
3 http://www.ucm.es/info/Astrof/software/reduceme/ reduceme.html

4 http://www.ing.iac.es/Astronomy/instruments/ids/
} 


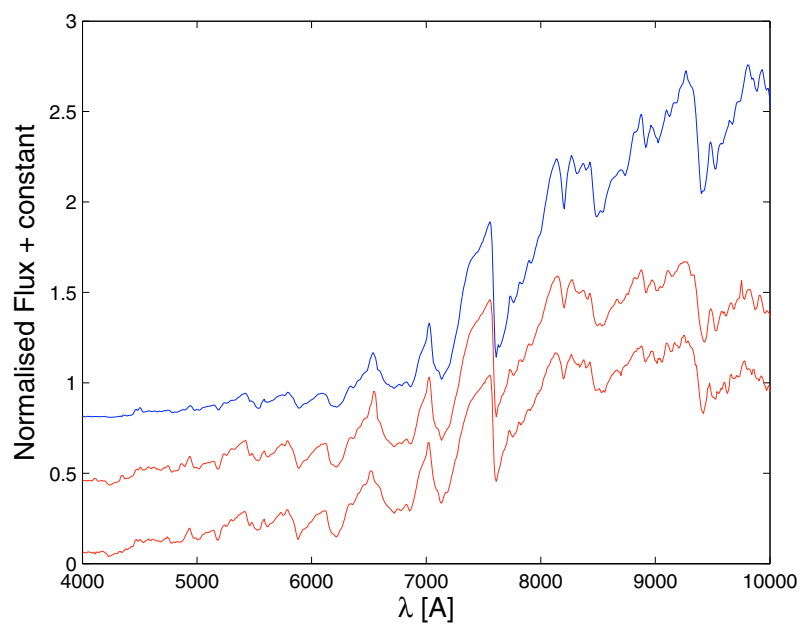

Fig. 1. CAFOS/2.2 m Calar Alto spectra of FL Vir AB (in blue), G 12515 , and G 125-14 (in red), from top to bottom. They are normalised at $7500 \AA$ and conveniently shifted in the vertical direction.

data were carried out using common tasks within the IRAF envirnonment. A part of the spectra of G 125-15, G 125-14, and GJ 1227 around the $\mathrm{H} \alpha$ region is shown in Fig. 2.

The $\mathrm{H} \alpha$ line in the intermediate-resolution spectrum of G 125-15 was in apparent, symmetric emission. We measured a pseudo-equivalent width of $\mathrm{p} E W(\mathrm{H} \alpha)=-5.8 \pm 0.7 \AA$. The line width at $10 \%$ height was $3.0 \AA$, significantly larger than those of arc lines or $\mathrm{H} \alpha$ emission lines in some active late-type stars observed during the run with the same instrumental configuration (of about $1.5 \AA$; Klutsch et al., in prep.). The absorption lines of G 125-15 appeared remarkably to be double, which implies that it is in turn a spectroscopic binary (SB2). The apparent broadening of the $\mathrm{H} \alpha$ line is more likely associated with the partial overlapping of two non-broadened emission lines, one redshifted and other blueshifted, than to a process of accretion from a circumstellar disc, such as those found in classical T Tauri stars. The consequences of the spectroscopic binarity of G 125-15 (hereafter G 125-15 AB) are discussed in Sect. 3. Besides this, we imposed a restrictive upper limit to the pseudo-equivalent width of the Li I $16707.8 \AA$ line. This is not surprising, since M dwarfs destroy their lithium content in 20-150 Ma. Similar upper limits were established for the $\mathrm{H} \alpha$ and $\mathrm{Li}$ I lines in $\mathrm{G}$ 125-14 (the $\mathrm{H} \alpha$ line of the secondary is filled or in very faint absorption). The results are summarised in Table 2.

Finally, we determined the radial heliocentric velocity of the three components in WDS 19312+3607. First, we analysed the cross-correlation functions of the IDS/INT spectra of $\mathrm{G} 125-15 \mathrm{AB}, \mathrm{G}$ 125-14, the comparison stars, and radialvelocity standard stars with the latest spectral types (about K7V) observed during our run. We found that the cross-correlation function of G 125-15 AB compared to any other single star observed with IDS had always two peaks, which is consistent with the primary having a double-lined spectrum and, hence, its spectroscopic binarity. We measured a radial velocity for G 125-14 with a reasonable precision of $2 \mathrm{~km} \mathrm{~s}^{-1}$ (Table 2), the binarity of G $125-15 \mathrm{AB}$ and the proximity between the two peaks in their cross-correlation functions allowed us to determine the radial velocities of $\mathrm{G} 125-15 \mathrm{~A}$ and $\mathrm{B}$ with a precision about three times lower. The difference in radial velocity between A and B was $40 \mathrm{~km} \mathrm{~s}^{-1}$ and the mean (i.e., the radial velocity of the barycentre

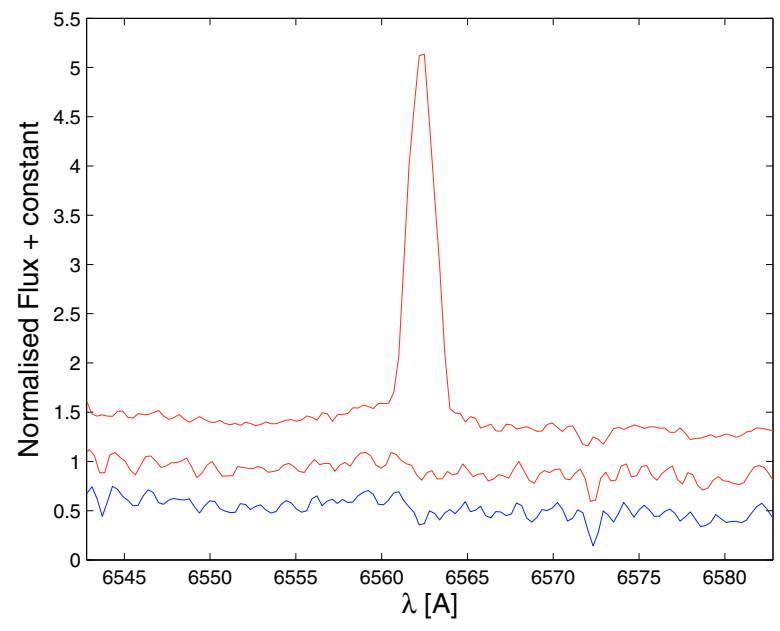

Fig. 2. A $40 \AA$-wide region around the $\mathrm{H} \alpha$ wavelength of the IDS/INT spectra of G 125-15, G 125-14 (in red), and GJ 1227 (in blue), from top to bottom. They are normalised at $6550 \AA$ and conveniently shifted in the vertical direction. Note the double lines in the spectra of G 125-15 and the resemblance between the spectra of G 125-14 and GJ 1227.

of $A$ and B) was consistent with the radial velocity of G 125-14 within the uncertainties. In practice, we were unable to differentiate between the components G 125-15 A and B because of the resemblance between the depths of the double lines in the spectrum and the heights of the two peaks in the cross-correlation functions. As a result, we assumed that A and B have the same basic parameters (e.g., mass, radius, effective temperature, magnitude, $\mathrm{H} \alpha$ emission).

\section{Discussion}

\subsection{Heliocentric distance}

Allen \& Reid (2008) derived $d=15.3_{-1.6}^{+1.9} \mathrm{pc}$ to $\mathrm{G} 125-15 \mathrm{AB}$ assuming singleness and normal radius and effective temperature (Reid et al. 2004, had derived $d=11.0 \pm 0.9 \mathrm{pc}$ to $\mathrm{G} 125-$ $15 \mathrm{AB}$, but also $d=85_{-15}^{+17}$ pc to G $125-14$ based on an incorrect $V$ magnitude). This implies a projected physical separation between G 125-15 AB and G 125-14 of $s=700_{-70}^{+90} \mathrm{AU}$.

There are numerous absolute magnitude-spectral type relations useful for determining heliocentric distances of intermediate- and late-M field dwarfs without parallax measurement (e.g., Henry et al. 1994; Hawley et al. 2002; Cruz et al. 2003; Phan-Bao \& Bessell 2006; Caballero et al. 2008). In this work, we used the $M_{J}$-Sp. type relation of Scholz et al. (2005), which is given in spectral type intervals of 0.5 dex. The derived absolute magnitude of $\mathrm{G} 125-14$ was $7.5_{-0.4}^{+0.3} \mathrm{mag}$. For the computation, we could only use the secondary $\mathrm{G} 125-14$ because the absolute magnitude of the primary G $125-15$ AB is affected by spectroscopic binary and activity (see Sect. 3.2). Using the value of $M_{J}$, the 2MASS $J$-band magnitude of G 125-14 in Table 2, and the Pogson law, $J-M_{J}=5 \log d-5$, and accounting for the scatter in the $M_{J}$-Sp. type relation, we estimated a heliocentric distance of $d=26_{-7}^{+12} \mathrm{pc}$. At this distance, the angular separation between $\mathrm{G} 125-15 \mathrm{AB}$ and $\mathrm{G}$ 125-14 translates into a projected physical separation of $s=1200_{-300}^{+600} \mathrm{AU}$, which ensures that WDS $19312+3607$ is one of the brightest, closest, low-mass systems with very low binding energies. 


\subsection{Close binarity and magnetic activity}

From Table 2, the primary is $1.0-1.5 \mathrm{mag}$ brighter than the secondary depending on the passband, while they have the same spectral type within a 0.5 dex uncertainty. The equal-mass binarity of the primary accounts for only about $0.75 \mathrm{mag}(2.5 \log 2)$. Since the stars are located at the same short heliocentric distance, the primary displays a wavelength-dependent overbrightness of $0.3-0.8 \mathrm{mag}$. In addition, $\mathrm{G} 125-15 \mathrm{AB}$ is redder than $\mathrm{G}$ 125-14. For example, the difference in $r-J$ colours, which depends strongly on the effective temperature, is $\Delta(r-J)=$ $0.77 \pm 0.03 \mathrm{mag}$. This deviation is marginally consistent within the 0.5 dex uncertainty in spectral type determination, but not with the observed overbrightness of $0.3-0.8 \mathrm{mag}$.

We estimated the ratios of effective temperatures and radii needed to explain the observed magnitude and colour differences between $\mathrm{G}$ 125-15 AB and $\mathrm{A} 125-14$. The ratio of the sum of observed fluxes at the $B$ to [8.0] bands is $\sum \lambda F_{\lambda,(1)} / \sum \lambda F_{\lambda,(2)} \sim$ 3.2 (using $\sum \lambda F_{\lambda}=\sum \frac{c}{\lambda} F_{\nu 0} 10^{-\frac{m_{\lambda}}{2.5}}$ and the corresponding zero-point conversion factors ${ }^{5}$ ), where "(1)" and "(2)" indicate G 125-15 $A B$ and $G$ 125-14, respectively. This quotient is a reasonable approximation to the ratio of total luminosities, $L_{(1)} / L_{(2)}$, from where one derives $2\left(R_{(1)} / R_{(2)}\right)^{2}\left(T_{\text {eff,(1) }} / T_{\text {eff,(2) }}\right)^{4} \sim$ 3.2 after assuming that the two components in $\mathrm{G} 125-15 \mathrm{AB}$ have the same mass and effective temperature. A cooler effective temperature, indicated by a redder $r-J$ colour, must be counterbalanced by a larger radius. We estimated that the two components in $\mathrm{G} 125-15 \mathrm{AB}$ are $\Delta T_{\text {eff }} \lesssim 5 \%$ cooler and $\Delta R \lesssim 30 \%$ larger than normal M4.5 dwarfs (including G 125-14), which have $T_{\text {eff }} \sim 2900-3300 \mathrm{~K}$ and $R \sim 0.23-0.26 R_{\odot}$. Effective temperature variations larger than $5 \%$ would lead to a different spectral type classification of G 125-15 AB and G 125-14.

Radii and effective temperatures in $\mathrm{M}$ dwarfs are affected by activity levels (Stauffer \& Hartmann 1986; Mullan \& MacDonald 2001; Torres \& Ribas 2002; López-Morales 2007; Reiners et al. 2007; Morales et al. 2008). According to Chabrier et al. (2007), the lower heat fluxes and, thus, larger radii and cooler effective temperatures of active low-mass stars and brown dwarfs compared to regular (inactive) stars are caused by the reduced convective efficiency, produced by the rapid rotation and high field strengths, and/or to magnetic spot coverage of the radiating surface. Previously, the activity scenario of G 125-15 AB was only consistent with the high relative X-ray flux (Daemgen et al. 2007; Allen \& Reid 2008). We now find that its is consistent with its $\mathrm{H} \alpha$ emission (Reid et al. 2004; this work), stellar expansion (by about 30\%; this work), and photometric variability (Hartman et al. 2004). This variability is more easily explained by an asymmetrical distribution of cool spots concentrated in certain hemispheres of two close, magnetically-active, orbitallocked, M4.5Ve stars rather than by pulsations in a low-mass dwarf. The period observed by Hartman et al. (2004) would be the rotational period of the system at $P_{\text {phot }} \sim 1.6 \mathrm{~d}$. This value is quite short for field $\mathrm{M}$ dwarfs and indicative of rapid rotation, as expected by the Chabrier et al. (2007) scenario.

\subsection{Space motion, age, mass, and semimajor axis}

We assumed that the strong magnetic activity in G $125-15 \mathrm{AB}$ is not due to youth, as previously understood, but to rapid rotation in a close orbital-locked system. First, if it were young, G 12514 should also display signposts of youth. Second, we derived

\footnotetext{
5 http://nsted.ipac. caltech. edu/NStED/docs/parhelp/ Photometry.html
}

Table 3. Properties of the WDS $19312+3607$ system.

\begin{tabular}{lcl}
\hline \hline Quantity & Value & Unit \\
\hline$\rho$ & $45.83 \pm 0.17$ & $\operatorname{arcsec}$ \\
& $0.764 \pm 0.003$ & $\operatorname{arcmin}$ \\
$\theta$ & $347.5 \pm 0.4$ & $\mathrm{deg}$ \\
$d$ & $26_{-7}^{+12}$ & $\mathrm{pc}$ \\
$\mu_{\alpha} \cos \delta$ & $-116.3 \pm 2.0$ & $\mathrm{mas} \mathrm{a}^{-1}$ \\
$\mu_{\delta}$ & $-100.6 \pm 1.2$ & $\mathrm{mas} \mathrm{a}^{-1}$ \\
$V_{r}$ & $-26 \pm 2$ & $\mathrm{~km} \mathrm{~s}^{-1}$ \\
$U$ & $+7 \pm 8$ & $\mathrm{~km} \mathrm{~s}^{-1}$ \\
$V$ & $-31 \pm 4$ & $\mathrm{~km} \mathrm{~s}^{-1}$ \\
$W$ & $+3 \pm 4$ & $\mathrm{~km} \mathrm{~s}^{-1}$ \\
$s$ & $1200_{-300}^{+600}$ & $\mathrm{AU}^{9}$ \\
$\tau$ & $0.6-5$ & $10^{9} \mathrm{a}$ \\
$\mathcal{M}_{\text {total }}$ & $0.54 \pm 0.09$ & $M_{\odot}$ \\
$U_{\mathrm{g}}$ & $-10 \pm 3$ & $10^{34} \mathrm{~J}$ \\
$P$ & 57 & $10^{3} \mathrm{a}$ \\
\hline
\end{tabular}

the Galactocentric space velocities $U V W$ of the WDS 19312+3607 system (Table 3) as in Montes et al. (2001). In the $U-V$ and $U-W$ diagrams, WDS $19312+3607$ lies outside the region that includes young moving groups with ages from $\tau \ll 100 \mathrm{Ma}$ (e.g., TW Hydra, $\beta$ Pictoris, AB Doradus) to $\tau \sim 300-600 \mathrm{Ma}$ (e.g., Castor, Hyades). However, the $U V W$ velocities of WDS $19312+3607$ are very different from those of old-disc stars (Leggett 1992). The most probable age of G 125-15 AB and G 125-14 from kinematics criteria is thus $\tau \sim 0.6-5 \mathrm{Ga}$.

We estimated the semimajor axis of the close binary G 125-15 AB assuming that the orbital period coincides with the photometric one. Before applying Kepler's third law, we had to estimate the masses of each component in the system from their absolute magnitudes and theoretical models. We determined the mass of $\mathrm{G} 125-14$, the only normal single dwarf in the system, at about $0.18 M_{\odot}$ using its $M_{J}$ magnitude (Table 3) and NextGen theoretical isochrones (Baraffe et al. 1998), which are only weakly sensitive to age if $0.3 \mathrm{Ga}<\tau<10 \mathrm{Ga}$. Based on the resemblance of spectral types, we cautiously assigned similar masses to the components in $\mathrm{G} 125-15 \mathrm{AB}$. Using these masses and the rotational-orbital period of the system, we estimated that the two stars are separated by only $0.019 \pm 0.004 \mathrm{AU}$ $\left(4.0 \pm 1.0 R_{\odot}\right.$ or about $10-20$ stellar radii).

The estimated semimajor axis $a$ is very short for M dwarfs and comparable to that of the well-known CM Dra system, which is formed by two population II M4.5 dwarfs (Lacy 1977; Vilhu et al. 1989; Chabrier \& Baraffe 1995; Metcalfe et al. 1996; Viti et al. 1997; Doyle et al. 2000; Morales et al. 2009). The two flaring stars in CM Dra are separated by $0.0175 \pm 0.0004 \mathrm{AU}$ and have a rapid tidally-synchronised rotation period of $1.27 \mathrm{~d}$, slightly shorter than the photometric period of G 125-15 AB. Because of its high inclination angle of $i=89.82 \mathrm{deg}$, CM Dra is an eclipsing binary. In analogy, the probability of eclipsing in $\mathrm{G} 125-15 \mathrm{AB}$ must be relatively high, at about $10-20 \%$ (as inferred from the ratio $R_{\star} / a$, where $R_{\star}$ is the radius of the two components). If the individual masses in G 125-15 AB were lower than expected for its spectral type due to activity (as seen in CM Dra - Lacy 1977), the semimajor axis would be shorter than 0.019 AU and the probability of eclipsing would increase.

Radii, masses, and effective temperatures of the two components in CM Dra, as well as in other eclipsing binaries, are widely used to compare observations to theoretical models. In contrast to CM Dra, which has a white-dwarf proper-motion 
companion at 26 arcsec, $\mathrm{G} 125-15 \mathrm{AB}$ has a wide proper-motion companion, G 125-14, that is a dwarf of the same spectral type within an uncertainty of $0.5 \mathrm{dex}$. The three stars can be used to study properly the relation between both stellar radius and effective temperature and activity at the bottom of the main sequence. As discussed in Sect. 3.2, G 125-15 AB and G 12514 also exhibit a temperature reversal with a relative amplitude of $\$ 5 \%$. These temperature reversals have been also detected in other cornerstone active M-type eclipsing binaries, such as the young brown-dwarf pair 2MASS J05352184-0546085 (Stassun et al. 2006, 2007).

\section{Summary}

Daemgen et al. (2007) and Allen \& Reid (2008) proposed that G $125-15$ is a single, active, M4.5Ve-type star in the solar neighbourhood younger than the Hyades $(\tau<600 \mathrm{Ma})$ based mainly on strong X-ray activity detected by Fuhrmeister \& Schmitt (2003). The dwarf is instead part of the wide binary system candidate WDS 19312+3607, which was tabulated earlier by Giclas et al. (1971). Its proper-motion companion candidate is G 12514, a poorly-known late-type dwarf more than 1.0 mag fainter located about 46 arcsec to the north. To test the youth and widebinarity hypotheses, we carried out spectroscopic, photometric, and astrometric analyses of the system using a collection of multiwavelength public and private data.

We found that the primary is a spectroscopic binary with $\mathrm{H} \alpha$ in broad emission and concluded that G 125-15 AB and G 12514 form a $0.6-5$ Ga-old hierarchical triple system at about $26 \mathrm{pc}$ from the Sun. The three components each have estimated masses of $0.18 M_{\odot}$. While G $125-15 \mathrm{AB}$ and G $125-14$ are separated by $\rho=45.83 \pm 0.17$ arcsec, which translates into a wide projected physical separation of $1200_{-300}^{+600} \mathrm{AU}, \mathrm{G} 125-15 \mathrm{~A}$ and $\mathrm{B}$ are separated by only about $0.02 \mathrm{AU}$. This close separation is responsible for the synchronisation of the pair and, thus, a short rotational period identical to the observed photometric period of $P_{\text {phot }}=1.6267 \mathrm{~d}$. Rapid rotation accounts for the higher magnetic activity of the pair, which is illustrated by its strong X-ray activity, $\mathrm{H} \alpha$ emission, photometric variability (possibly associated with the presence of cool spots), and, in particular, larger radii of the two components, with respect to normal dwarfs of the same spectral type.

The brightness and proximity of WDS $19312+3607$ will facilitate astrometric, photometric, and spectroscopic followup studies, particularly those designed to determine accurate trigonometric parallax, age, and radial and rotational velocities of the system, and investigate the relation between radius, effective temperature, and magnetic activity.

Acknowledgements. We thank the anonymous referee for helpful comments and P. G. Pérez-González for software help. Based on observations collected at the Centro Astronómico Hispano Alemán (CAHA) at Calar Alto, operated jointly by the Max-Planck Institut für Astronomie and the Instituto de Astrofísica de Andalucía. Based on observations made with the Isaac Newton Telescope operated on the island of La Palma by the Isaac Newton Group in the Spanish Observatorio del Roque de los Muchachos of the Instituto de Astrofísica de Canarias. This research made use of the SIMBAD, operated at Centre de Données astronomiques de Strasbourg, France, and NASA's Astrophysics Data System. Financial support was provided by the Universidad Complutense de Madrid, the Comunidad Autónoma de Madrid, and the Spanish Ministerio de
Ciencia e Innovación under grants AyA2008-00695, AyA2008-06423-C03-03, and SP2009/ESP-1496.

\section{References}

Abazajian, K. N., Adelman-McCarthy, J. K., Agüeros, M. A., et al. 2009, ApJS, 182,543

Allen, P. R., \& Reid, I. N. 2008, AJ, 135, 2024

Artigau, É., Lafrenière, D., Doyon, R., et al. 2007, ApJ, 659, L49

Baraffe, I., Chabrier, G., Allard, F., \& Hauschildt, P. H. 1998, A\&A, 337, 403

Caballero, J. A. 2007, A\&A, 462, L61

Caballero, J. A. 2009, A\&A, 507, 251

Caballero, J. A. 2010, A\&A, 514, A18

Caballero, J. A., Burgasser, A. J., \& Klement, R. 2008, A\&A, 488, 181

Caballero, J. A., Miret, F. X., Genebriera, J., et al. 2010, in Highlights of Spanish Astrophysics V, Astrophysics and Space Science Proceedings (Berlin, Heidelberg: Springer-Verlag), 379

Cardiel, N. 1999, Ph.D. Thesis, Universidad Complutense de Madrid, Spain

Chabrier, G., \& Baraffe, I. 1995, ApJ, 451, L29

Chabrier, G., Gallardo, J., \& Baraffe, I. 2007, A\&A, 472, L17

Cruz, K. L., Reid, I. N., Liebert, J., Kirkpatrick, J. D., \& Lowrance, P. J. 2003, AJ, 126, 2421

Daemgen, S., Siegler, N., Reid, I. N., \& Close, L. M. 2007, ApJ, 654, 558

Doyle, L. R., Deeg, H. J., Kozhevnikov, V. P., et al. 2000, ApJ, 535, 338

Fuhrmeister, B., \& Schmitt, J. H. M. M. 2003, A\&A, 403, 247

Giclas, H. L., Burnham, R., \& Thomas, N. G. 1971, Lowell proper motion survey Northern Hemisphere, The G numbered stars, 8991 stars fainter than magnitude 8 with motions $>0$ '. 26/year, Flagstaff, Arizona, Lowell Observatory

Hambly, N. C., MacGillivray, H. T., Read, M. A., et al. 2001, MNRAS, 326, 1279

Hanson, R. B., Klemola, A. R., Jones, B. F., \& Monet, D. G. 2004, AJ, 128, 1430

Hartman, J. D., Bakos, G., Stanek, K. Z., \& Noyes, R. W. 2004, AJ, 128, 1761

Hawley, S. L., Covey, K. R., \& Knapp, G. R. 2002, AJ, 123, 3409

Henry, T. J., Kirkpatrick, J. D., \& Simons, D. A. 1994, AJ, 108, 1437

Høg, E., Fabricius, C., Makarov, V. V., et al. 2000, A\&A, 355, L27

Ivanov, G. A. 2008, KFNT, 24, 480, VizieR on-line data catalogue: I/306A

Joy, A. H. 1947, ApJ, 105, 96

Lacy, C. H. 1977, ApJS, 34, 479

Leggett, S. K. 1992, ApJS, 82, 351

Lépine, S., \& Shara, M. M. 2005, AJ, 129, 1483

López-Morales, M. 2007, ApJ, 660, 732

Luyten, W. J. 1979, LHS catalogue. A catalogue of stars with proper motions exceeding 0.'5 annually, Minneapolis, University of Minnesota, 2nd edn.

Martín, E. L., Delfosse, X., Basri, G., et al. 1999, AJ, 118, 2466

Metcalfe, T. S., Mathieu, R. D., Latham, D. W., \& Torres, G. 1996, ApJ, 456, 356

Montes, D., López-Santiago, J., \& Gálvez, M. C. 2001, MNRAS, 328, 45

Morales, J. C., Ribas, I., \& Jordi, C. 2008, A\&A, 478, 507

Morales, J. C., Ribas, I., Jordi, C., et al. 2009, ApJ, 691, 1400

Muiños, J. L. 2006, On behalf of the Carlsberg Meridian Catalog Number 14 team, VizieR on-line catalogue I/304

Mullan, D. J., \& MacDonald, J. 2001, ApJ, 559, 353

Phan-Bao, N., \& Bessell, M. S. 2006, A\&A, 446, 515

Radigan, J., Lafrenière, D., Jayawardhana, R., \& Doyon, R. 2009, ApJ, 698, 405

Reid, I. N., Cruz, K. L., Allen, P., et al. 2004, AJ, 128, 463

Reiners, A., Seifahrt, A., Stassun, K. G., Melo, C., \& Mathieu, R. D. 2007, ApJ, 671, L149

Salim, S., \& Gould, A. 2003, ApJ, 582, 1011

Scholz, R.-D., Meusinger, H., \& Jahrei $\beta$, H. 2005, A\&A, 442, 211

Skrutskie, M. F., Cutri, R. M., Stiening, R., et al. 2006, AJ, 131, 1163

Stassun, K. G., Mathieu, R. D., \& Valenti, J. A. 2006, Nature, 440, 311

Stassun, K. G., Mathieu, R. D., \& Valenti, J. A. 2007, ApJ, 664, 1154

Stauffer, J. R., \& Hartmann, L. W. 1986, ApJS, 81, 531

Torres, G., \& Ribas, I. 2002, ApJ, 567, 1140

Vilhu, O., Ambruster, C. W., Neff, J. E., et al. 1989, A\&A, 222, 179

Viti, S., Jones, H. R. A., Schweitzer, A., et al. 1997, MNRAS, 291, 780

Weis, E. W. 1996, AJ, 112, 2300

Whitworth, A. P., \& Stamatellos, D. 2006, A\&A, 458, 817

Worley, C. E., \& Douglass, G. G. 1997, A\&AS, 125, 523 